\title{
Analysis of Adult Patients with Tinea Capitis in Southeastern Korea
}

\author{
Hyun Ji Lee, Jun Young Kim, Kyung Duck Park, Yong Hyun Jang, Seok-Jong Lee, Yong Jun Bang ${ }^{1}$, \\ Jae Bok Jun ${ }^{1}$, Weon Ju Lee \\ Department of Dermatology, School of Medicine, Kyungpook National University, Kyungpook National University Hospital, \\ ${ }^{1}$ Institute of Medical Mycology, Catholic Skin Clinic, Daegu, Korea
}

Background: Tinea capitis is a cutaneous infection of dermatophytes and predominant in children. Although tinea capitis in Korea is controlled by oral antifungal medications and concerted public health initiatives, it's still a health issue. Objective: To investigate changes in the epidemiological and mycological characteristics of adult patients with tinea capitis in southeastern Korea. Methods: Using medical records from Kyungpook National University Hospital and Catholic Skin Clinic from 1989 to 2018, we retrospectively investigated the epidemiological and mycological characteristics of 266 adult patients (aged over 20) with tinea capitis. Results: Among total 266 patients, 239 were $\mathrm{KOH}$-positive. The annual incidence of tinea capitis ranged from 3 to 18 between 1989 and 2018. Of the total, 54 (20.30\%) were male and $212(79.70 \%)$ were female. Eighty patients $(30.08 \%)$ were in their seventies, the most commonly affected age group. Of the remaining, $58(21.80 \%)$ were in their sixties, and 41 (15.41\%) in eighties. Among all, 77 (28.95\%) visited the hospital in summer, $72(27.07 \%)$ in spring, $64(24.06 \%)$ in winter, and $53(19.92 \%)$ in fall. Dermatophytes were cultured from 171 patients. Microsporum canis was the most common dermatophyte $(42.48 \%)$, while Trichophyton ru-

Received August 22, 2019, Revised November 24, 2019, Accepted for publication November 26, 2019

Corresponding author: Weon Ju Lee, Department of Dermatology, Kyungpook National University Hospital, 130 Dongdeok-ro, Jung-gu, Daegu 41944, Korea. Tel: 82-53-420-5838, Fax: 82-53-426-0770, E-mail: weonju@knu. ac. kr

ORCID: https://orcid.org/0000-0001-5708-1305

This is an Open Access article distributed under the terms of the Creative Commons Attribution Non-Commercial License (http://creativecommons. org/licenses/by-nc/4.0) which permits unrestricted non-commercial use, distribution, and reproduction in any medium, provided the original work is properly cited.

Copyright (c) The Korean Dermatological Association and The Korean Society for Investigative Dermatology brum was the second (15.79\%). Of the 266 patients, 186 $(69.92 \%)$ lived in urban areas and $80(30.08 \%)$ in rural areas. Conclusion: The epidemiological and mycological characteristics of adult patients with tinea capitis were different from those of children in terms of annual incidence, sex distribution, and isolated dermatophytes. These results provide useful information for the treatment and prevention of tinea capitis. (Ann Dermatol 32(2) 109 114, 2020)

\section{-Keywords-}

Dermatophytosis, Epidemiology, Microsporum canis, Tinea capitis

\section{INTRODUCTION}

Tinea capitis is an infectious skin disease caused by dermatophytes that is found primarily in children ${ }^{1}$ and more commonly in males. Its clinical manifestation can range from small scaly patches to large inflammatory pustular plaques with extensive hair loss. Immunosuppressive conditions, such as diabetes mellitus and prolonged steroid medication, have also been associated with tinea capitis ${ }^{2}$. The epidemiological and mycological characteristics of tinea capitis change over time and are influenced by environmental conditions, socioeconomic status, and individual immunity. Microsporum ferrugineum was the most common dermatophyte responsible for tinea capitis in Korea in the $1960 \mathrm{~s}^{3}$, but Microsporum canis has increasingly been identified as the cause of tinea capitis in Korea since the $1970 \mathrm{~s}^{4}$. Although tinea capitis can be controlled with oral antifungal medication and public health campaigns, it remains a public health issue in Korea ${ }^{5}$. In particular, the incidence of tinea capitis is increasing in 


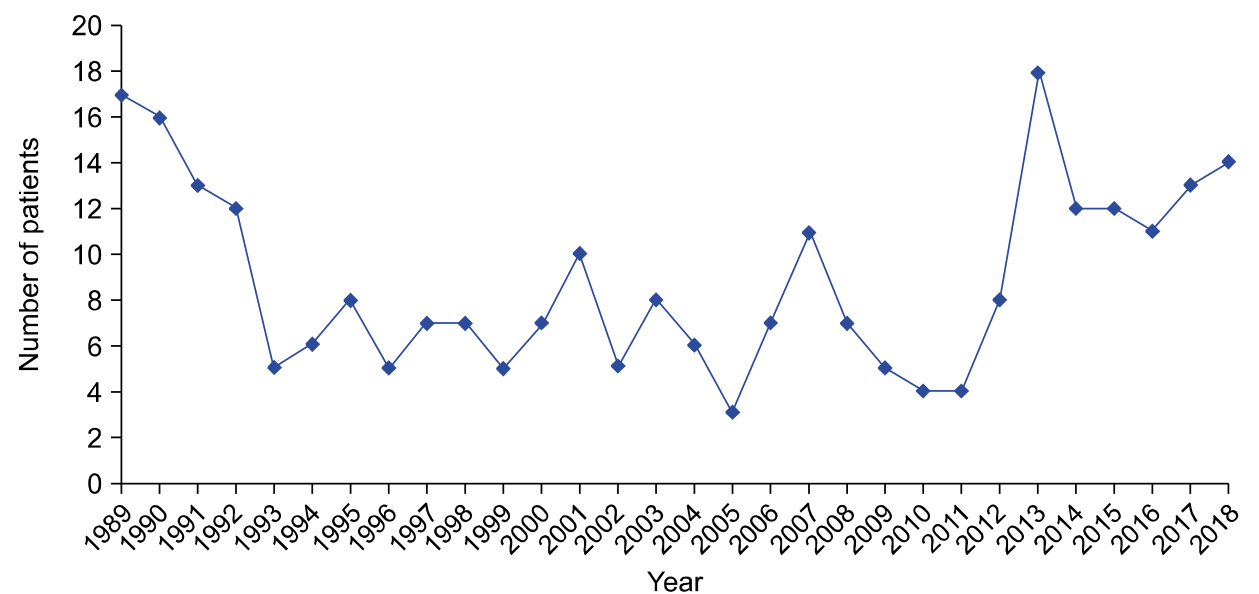

Fig. 1. Annual incidence for adult patients with tinea capitis.

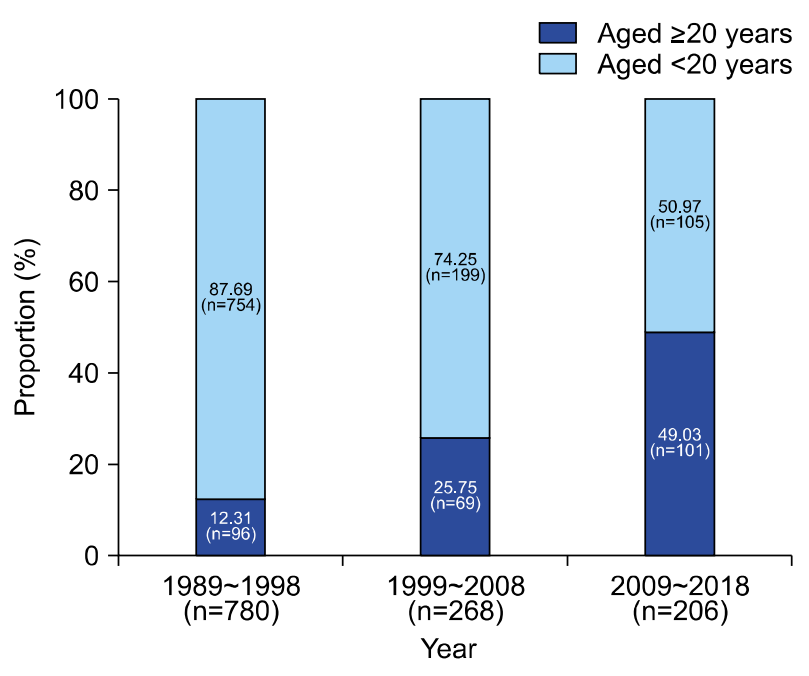

Fig. 2. Proportion of adult patients in total tinea capitis incidence by time period. The proportion of tinea capitis patients aged over 20 years has been increasing with time: $12.31 \%$ between 1989 and 1998, 25.75\% between 1999 and 2008, and 49.03\% between 2009 and 2018.

adults, even though it is decreasing in children. We thus investigated changes in the epidemiological and mycological characteristics of adult patients with tinea capitis in southeastern Korea.

\section{MATERIALS AND METHODS}

\section{Patients}

Using medical records from Kyungpook National University Hospital and the Catholic Skin Clinic from 1989 to 2018, we retrospectively investigated the epidemiological and mycological characteristics of 266 patients aged over 20 years out of 1,254 patients with tinea capitis.

\section{Diagnosis of dermatophyte infection}

Using their medical records, the information from 266 adult patients with tinea capitis was retrospectively analyzed to determine the annual incidence, age distribution, sex distribution, seasonal incidence, identity of the isolated dermatophytes, and residence. Scales and hairs were obtained from the scalp for fungal examination, and analysis with $15 \% \mathrm{KOH}$ was conducted. Of the 266 patients, $239(89.85 \%)$ were found to be $\mathrm{KOH}$-positive. The dermatophytes responsible for the infection were isolated from fungal cultures using potato-dextrose agar and cornmeal Tween 80 media. The cultures were kept at $24^{\circ} \mathrm{C} \sim 26^{\circ} \mathrm{C}$ and examined after 2 to 4 weeks. Dermatophytes were cultured from 171 patients, and microscopic examination was conducted after staining with lactophenol cotton blue to identify fungal hyphae with conidia. Wood lamp tests were also used to detect fluorescent fungal elements.

\section{Ethics statements}

We conducted this study after approval by the Institutional Review Board of Kyungpook National University Hospital (IRB no. 2019-03-014). Informed patient consent was waived by the board.

\section{RESULTS}

\section{Annual incidence}

The annual incidence of patients aged over 20 years with tinea capitis ranged from 3 to 18 between 1989 and 2018 (Fig. 1), with a mean annual incidence rate of 8.9 . In terms of the change in the mean incidence rate over time, it was 9.6 between 1989 and 1998 (96 patients), 6.9 between 1999 and 2008 (69 patients), and 10.1 between 2009 and 2018 (101 patients). The annual incidence of tinea capitis in patients aged over 20 years was lowest in 2005 and highest in 2013. Fig. 2 presents the proportion of tinea 
capitis patients that were over 20 years old; of the total of 780 patients diagnosed with tinea capitis between 1989 and 1998, 96 were older than 20 (12.31\%), compared to 69 out of 268 between 1999 and 2008 (25.75\%), and 101 out of 206 between 2009 and 2018 (49.03\%) for a total of 266 out of 1,254 for the entire study period (21.21\%).

\section{Age distribution}

Table 1 presents the incidence of tinea capitis by age group. From 1989 to 2018, the most commonly affected age group were those patients in their seventies, with 80 patients diagnosed $(30.08 \%)$. Of the remaining patients, $58(21.80 \%)$ were in their sixties, and $41(15.41 \%)$ were in their eighties. The rest were in their fifties, forties, thirties, twenties, and nineties in order. This pattern was similar for the individual 1989 1998, 1999 2008, and 2009 2018 subperiods, though some differences were observed. From 1989 to 1998 , the most commonly affected age group was those patients in their sixties, with 30 patients diagnosed $(31.25 \%)$. Of the remaining patients, $20(20.83 \%)$ were in their seventies, 18 (18.75\%) were in their fifties, and the rest were in thirties, forties, eighties, and twenties in order. From 1999 to 2008, 19 patients $(27.54 \%)$ were respectively in their sixties and seventies, 10 (14.49\%) were in their fifties. The rest were in their eighties, forties, twenties, thirties, and nineties in order, each with 6 patients or fewer. From 2009 to 2018, 41 patients $(40.59 \%)$ were in their seventies, $29(28.71 \%)$ were in their eighties, $12(11.88 \%)$ were in their fifties, and the rest were in their sixties, twenties, forties, nineties, and thirties in order.

\section{Sex distribution}

For the entire study period (1989 2018), 54 of the 266

Table 1. Age distribution of adult patients with tinea capitis

\begin{tabular}{|c|c|c|c|c|c|c|c|c|c|}
\hline \multirow{2}{*}{ Period } & \multicolumn{8}{|c|}{ Age (yr) } & \multirow{2}{*}{ Total } \\
\hline & $20 \sim 29$ & $30 \sim 39$ & $40 \sim 49$ & $50 \sim 59$ & $60 \sim 69$ & $70 \sim 79$ & $80 \sim 89$ & $90 \sim 99$ & \\
\hline $1989 \sim 1998$ & $3(3.13)$ & $10(10.42)$ & $9(9.38)$ & $18(18.75)$ & $30(31.25)$ & $20(20.83)$ & $6(6.25)$ & $0(0.00)$ & 96 (100) \\
\hline $1999 \sim 2008$ & $3(4.35)$ & $4(5.80)$ & $5(7.25)$ & $10(14.49)$ & $19(27.54)$ & 19 (27.54) & $6(8.70)$ & $3(4.35)$ & 69 (100) \\
\hline $2009 \sim 2018$ & 5 (4.95) & 1 (0.99) & 2 (1.98) & 12 (11.88) & 9 (8.91) & 41 (40.59) & $29(28.71)$ & 2 (1.98) & 101 (100) \\
\hline Total & $11(4.14)$ & $15(5.64)$ & $16(6.02)$ & $40(15.04)$ & $58(21.80)$ & $80(30.08)$ & $41 \quad(15.41)$ & $5(1.88)$ & $266(100)$ \\
\hline
\end{tabular}

Values are presented as number (\%).

Table 2. Seasonal distribution of adult patients with tinea capitis

\begin{tabular}{|c|c|c|c|c|c|}
\hline \multirow{2}{*}{ Period } & \multicolumn{4}{|c|}{ Season } & \multirow{2}{*}{ Total } \\
\hline & Spring & Summer & Fall & Winter & \\
\hline $1989 \sim 1998$ & $31(32.29)$ & $28(29.17)$ & $13(13.54)$ & $24(25.00)$ & 96 (100) \\
\hline $1999 \sim 2008$ & $18(26.09)$ & $23(33.33)$ & $8(11.59)$ & $20(28.99)$ & 69 (100) \\
\hline $2009 \sim 2018$ & $23(22.77)$ & $26(25.74)$ & 32 (31.68) & $20(19.80)$ & $101(100)$ \\
\hline Total & $72(27.07)$ & 77 (28.95) & $53(19.92)$ & $64(24.06)$ & $266(100)$ \\
\hline
\end{tabular}

Values are presented as number (\%).

Table 3. Dermatophytes isolated from adult patients with tinea capitis

\begin{tabular}{|c|c|c|c|c|c|c|c|c|c|}
\hline \multirow[b]{2}{*}{ Period } & \multicolumn{8}{|c|}{ Causative fungus } & \multirow[b]{2}{*}{ Total } \\
\hline & $\begin{array}{c}\text { Micros- } \\
\text { porum } \\
\text { canis }\end{array}$ & $\begin{array}{l}\text { Tricho- } \\
\text { phyton } \\
\text { rubrum }\end{array}$ & $\begin{array}{c}\text { Tricho- } \\
\text { phyton } \\
\text { menta- } \\
\text { grophytes }\end{array}$ & $\begin{array}{c}\text { Tricho- } \\
\text { phyton } \\
\text { verrucosum }\end{array}$ & $\begin{array}{c}\text { Tricho- } \\
\text { phyton } \\
\text { tonsurans }\end{array}$ & $\begin{array}{l}\text { Micros- } \\
\text { porum } \\
\text { gypseum }\end{array}$ & $\begin{array}{c}\text { Contami- } \\
\text { nant }\end{array}$ & Negative & \\
\hline $1989 \sim 1998$ & $41(42.71)$ & $14(14.58)$ & $3(3.13)$ & $3(3.13)$ & $1(1.04)$ & $0(0.00)$ & $18(18.75)$ & $16(16.67)$ & $96(100)$ \\
\hline $1999 \sim 2008$ & $28(40.58)$ & 7 (10.14) & $1(1.45)$ & $2(2.90)$ & $2(2.90)$ & $0(0.00)$ & $10(14.49)$ & $19(27.54)$ & 69 (100) \\
\hline $2009 \sim 2018$ & $44(43.56)$ & $21(20.79)$ & $3(2.97)$ & $0(0.00)$ & $0(0.00)$ & $1(0.99)$ & $9(8.91)$ & $23(22.77)$ & $101(100)$ \\
\hline Total & $113(42.48)$ & $42(15.79)$ & $7(2.63)$ & $5(1.88)$ & $3(1.13)$ & $1(0.38)$ & $37(13.91)$ & $58(21.80)$ & $266(100)$ \\
\hline
\end{tabular}

Values are presented as number (\%). 
Table 4. Dermatophytosis coexisting with tinea capitis

\begin{tabular}{lc}
\hline Coexisting dermatophytosis & No. of patients $(\%)$ \\
\hline Tinea faciei & $22(8.27)$ \\
Tinea corporis & $16(6.02)$ \\
Tinea manus & $1(0.38)$ \\
Tinea cruris & $2(0.75)$ \\
Tinea pedis & $7(2.63)$ \\
Tinea unguium & $28(10.53)$ \\
\hline
\end{tabular}

patients $(20.30 \%)$ were male and the remaining 212 patients $(79.70 \%)$ were female, a ratio of $1: 3.9$. In terms of the subperiods, from 1989 to 1998,17 of the 96 patients $(17.71 \%)$ were male and $79(82.29 \%)$ were female (1:4.6), while from 1999 to 2008,17 of the 69 patients $(24.64 \%)$ were male and $52(75.36 \%)$ were female (1:3.1). Finally, from 2009 to 2018,20 of the 101 patients (19.80\%) were male and $81(80.20 \%)$ were female $(1: 4.1)$.

\section{Seasonal distribution}

Table 2 shows that, from 1989 to 2018,77 of the 266 patients $(28.95 \%)$ visited hospital in summer, $72(27.07 \%)$ in spring, 64 (24.06\%) in winter, and $53(19.92 \%)$ in fall. In the earlier subperiod (1989 1998), spring was the most common season for patients to visit hospital. From 1999 to 2008, summer was the most common season, and from 2009 to 2018, fall was the most common season.

\section{Isolation of dermatophytes}

As can be seen in Table 3, from 1989 to 2018, M. canis was the most common dermatophyte $(42.48 \%)$ isolated from tinea capitis patients, while Trichophyton rubrum was the second most common (15.79\%). From 1989 to 1998, M. canis was the most common dermatophyte $(42.71 \%)$, with $T$. rubrum the second most common (14.58\%). From 1999 to 2008, M. canis was also the most common dermatophyte $(40.58 \%)$, followed by T. rubrum at $10.14 \%$. From 2009 to 2018, M. canis was the most common dermatophyte $(43.56 \%)$ isolated from tinea capitis and $T$. rubrum was the second most common $(20.79 \%)$.

\section{Patient residence}

Residence was divided based on Korean administrative district. For the entire study period, 186 of the 266 patients $(69.92 \%)$ lived in urban areas and 80 (30.08\%) lived in rural areas. From 1989 to 1998, 65 of the 96 patients $(67.71 \%)$ lived in urban areas and 31 (32.29\%) in rural areas. From 1999 to 2008, 56 of the 69 patients (81.16\%) lived in urban areas and $13(18.84 \%)$ lived in rural areas. From 2009 to 2018 , 65 of the 101 patients $(64.36 \%$ ) lived in urban areas and 36 (35.64\%) lived in rural areas.

\section{Coexisting dermatophytosis}

A total of 101 patients from 2009 to 2018 were investigated for topographical distribution coexisting with tinea capitis (Table 4). Tinea faciei coexisted with tinea capitis in 22 patients $(8.27 \%)$ and tinea corporis coexisted in 16 patients $(6.02 \%)$. Interestingly, tinea unguium coexisted in 28 patients $(10.53 \%)$.

\section{Clinical symptoms}

A total of 101 patients from 2009 to 2018 presented with scaly hairless erythematous patches on the scalp. Ten patients exhibited kerion celsi, and 12 patients were misdiagnosed with seborrheic dermatitis.

\section{Causative agents and underlying diseases}

Of the 101 patients diagnosed with tinea capitis from 2009 to 2018, 9 had past contact history with cats. Some patients had underlying diseases; of these, 11 had diabetes mellitus, 28 had hypertension, and 13 had other underlying diseases.

\section{Treatment of tinea capitis}

From 2009 to 2018, all of the patients were treated with oral antifungal medicines such as terbinafine and itraconazole: 71 patients $(70.30 \%)$ were treated with terbinafine, $25(24.75 \%)$ with itraconazole, and 5 (4.95\%) with both. All of them were treated $1 \sim 3$ months, and then positive results in follow up $\mathrm{KOH}$ test prolonged the treatment duration. In the end, 60 patients were cured, 25 patients were lost during follow up period, and 2 patients were failed.

\section{Comparison between patients infected with $M$. canis and $T$. rubrum}

From 2009 to 2018, 41 patients were infected with $M$. canis and 21 patients with T. rubrum. M. canis infection was highest in their seventies (20 patients) and T. rubrum infection in their eighties (11 patients). M. canis infection was most common in spring (15 patients) and T. rubrum infection in winter (8 patients). M. canis infection developed kerion celsi but T. rubrum infection did not. Contact history of cats or other animals was noted only in $M$. canis infection. The ratio of cure to loss of follow up or failure during antifungal treatment was much higher in $M$. canis infection than in T. rubrum Infection.

\section{DISCUSSION}

Our previous study reported that a total of 2,029 patients 
developed tinea capitis between 1978 and 1984. The number of adult patients with tinea capitis was only 41 $(2.0 \%)$ in that study. However, the present study reveals that the annual incidence of tinea capitis in adults has increased over time from $41(1978 \sim 1988)$ to 101 patients (2009 2018). In addition, the proportion of patients aged over 20 years from 1989 to 1998 was $12.31 \%$, increasing to $25.75 \%$ from 1999 to 2008 and to $49.03 \%$ from 2009 to 2018. Compared with the proportion from 1978 to 1988, the proportion of adults with tinea capitis from 2009 to 2018 has thus increased tremendously. However, Cheon et al. ${ }^{6}$ reported that the incidence of tinea capitis from 2016 to 2017 was still higher in children below 10 years old, while Kim et al. ${ }^{7}$, Shin et al. ${ }^{8}$, Chun et al. ${ }^{9}$, and Kim et al. ${ }^{10}$ also found that children below 10 years old were the most common patient group for tinea capitis. The hygiene of children has improved significantly compared to the past, so the incidence of tinea capitis has been lowered. However, the life patterns of adults have not changed and the life span increased. It explains increased incidence of adult patients with tinea capitis. In addition, the increasing incidence of adult tinea capitis may be attributed to the underlying diseases, such as diabetes, increased use of steroids and immunosuppressants, and the reduction of triglycerides with antifungal effects ${ }^{4,7}$. Our previous study found that 34 of the 41 adult tinea capitis patients were female. In this study, 54 of the 266 patients $(20.30 \%)$ from 1989 to 2018 were male and the remaining 212 patients $(79.70 \%)$ were female, while 20 of the 101 patients $(19.80 \%)$ from 2009 to 2018 were male and $81(80.20 \%)$ were female. Thus, there was a similar sex distribution for the disease across all of the subperiods. Kim et al. ${ }^{7}$, Shin et al. ${ }^{8}$, and Kim et al. ${ }^{10}$ also reported that the incidence of tinea capitis was higher in adult females. In contrast, Chun et al. ${ }^{9}$ found that the incidence of tinea capitis was higher in adult males. In addition, among children with tinea capitis, male patients are more common. In this study, female predominance in incidence of tinea capitis may be explained by hospital friendly tendency and prolonged life span. In addition, keeping pets and contact with them may be more frequent in female than in male.

Our previous study revealed that patients in their sixties had the highest incidence of tinea capitis ${ }^{4}$. Similarly, in the present study, patients in their sixties were the most commonly inflicted age group from 1989 to 1998. However, from 2009 to 2018, the highest incidence rate was among patients in their seventies.

In a previous study, it was reported that patients with tinea capitis were diagnosed most commonly in winter ${ }^{3}$. Kim et al. ${ }^{7}$, Shin et al. ${ }^{8}$, and Chun et al. ${ }^{9}$ also reported that winter was the most common season for tinea capitis occurrence. However, patients with tinea capitis in the present study most commonly visited hospital in summer from 1989 to 2018, and from 2009 to 2018, patients with tinea capitis most commonly visited hospital in fall. Cutaneous fungal infections usually occur in a hot and humid weather. Therefore, incidence of the fungal infection may be highest in summer ${ }^{11}$. However, incidence of tinea capitis can be influenced by changes in life style and accessibility to hospitals.

In our previous study, M. canis (36 patients, $87.80 \%$ ), Trichophyton verrucosum (2 patients), Trichophyton mentagrophytes (1 patient), Trichophyton ferrugineum (1 patient), and $T$. rubrum (1 patient) were found to be the causal agents for tinea capitis ${ }^{4}$. In the present study, $M$. canis was the most common dermatophyte $(43.56 \%)$ isolated from tinea capitis from 2009 to 2018, followed by $T$. rubrum $(20.79 \%)$, both of which were also the two most common dermatophytes for the entire study period $\left(42.48 \%\right.$ and $15.79 \%$, respectively). Cheon et al. ${ }^{6}$ also reported that the second most common causative agent for tinea capitis was T. rubrum. In addition, Kim et al. ${ }^{7}$ and Shin et al. $^{8}$ reported the same results, though the proportion of $T$. rubrum was lower in these studies compared to the present study. Chun et al. ${ }^{9}$ reported that $T$. mentagrophytes was the second most common causative agent for tinea capitis. Dermatophytes evolve along with socioeconomic conditions, population mobility and changes in human life style ${ }^{12}$. T. verrucosum is a frequent cause of dermatophytosis of cattle and other farm animals. Public health care and improved environmental conditions might lead to a decrease in the incidence of tinea capitis caused by $T$. verrucosum. Trichophyton tonsurans is a frequent cause of tinea capitis in teenagers, especially gladiators, such as judo players and wrestlers ${ }^{13}$. Although tinea capitis caused by $T$. tonsurans has spread to non-gladiators since 2000, it is still not common causative agent in adults. T. rubrum is an anthropophilic dermatophyte and has been prevailing in Korea, constituting more than $90 \%$ of dermatophytoses since $1991^{11}$.

In our previous study, tinea corporis (3 patients), tinea faciei ( 2 patients), and tinea pedis ( 1 patient) were found to coexist with tinea capitis ${ }^{4}$. In the present study, from 2009 to 2018 , tinea unguium (28 patients), tinea faciei (22 patients), tinea corporis (16 patients), tinea pedis (7 patients), tinea manus ( 1 patient), and tinea cruris (2 patients) coexisted with tinea capitis. These results differ from those reported by previous studies. For example, Kim et al. ${ }^{7}$ and Shin et al. $^{8}$ reported that tinea faciei was the most common coexisting dermatophytosis.

Our previous study identified contact with cats (11 pa- 
tients) and cows ( 2 patients) as causative factors ${ }^{4}$. In the present study, of the 101 patients from 2009 to 2018, 9 had past contact history with cats. Some patients also had diabetes mellitus (11 patients), hypertension (28 patients), and other underlying diseases (13 patients). Kim et al. ${ }^{7}$ and Shin et al. ${ }^{8}$ reported that cats and dogs were the main causative factors for tinea capitis and that adult patients exhibited underlying diseases.

Griseofulvin was used as a therapeutic medication for tinea capitis before the introduction of new antifungal medicines such as terbinafine and itraconazole. Terbinafine is an effective agent in the treatment of tinea capitis because of its shorter treatment schedule and lower recurrence rate $^{14}$. Itraconazole is as effective as terbinafine in the treatment of tinea capitis ${ }^{15-17}$, while ketoconazole should be avoided due to severe side effects ${ }^{18}$. In the present study, the tinea capitis cases were treated effectively with oral terbinafine or itraconazole.

In summary, the epidemiological and mycological characteristics of adult patients with tinea capitis differ from those of children with tinea capitis in terms of annual incidence, sex distribution, and isolated dermatophytes. In particular, tinea capitis in adult patients showed increasing annual incidence, female predominance, and the increased proportion of $T$. rubrum among causative dermatophytes. This represents potentially useful information for the treatment and prevention of tinea capitis.

\section{CONFLICTS OF INTEREST}

The authors have nothing to disclose.

\section{ORCID}

Hyun Ji Lee, https://orcid.org/0000-0002-4222-1835

Jun Young Kim, https://orcid.org/0000-0002-2999-1018

Kyung Duck Park, https://orcid.org/0000-0002-6067-7262

Yong Hyun Jang, https://orcid.org/0000-0003-1706-007X

Seok-Jong Lee, https://orcid.org/0000-0002-6131-632X

Yong Jun Bang, https://orcid.org/0000-0001-7242-9306

Jae Bok Jun, https://orcid.org/0000-0003-4834-4526

Weon Ju Lee, https://orcid.org/0000-0001-5708-1305

\section{REFERENCES}

1. Michaels BD, Del Rosso JQ. Tinea capitis in infants: recognition, evaluation, and management suggestions. J Clin Aesthet Dermatol 2012;5:49-59.
2. Hay RJ. Tinea capitis: current status. Mycopathologia 2017; 182:87-93.

3. Hong JK. A supplementary study on tinea capitis in Taegu city. Korean J Dermatol 1962;2:7-14.

4. Oh SH, Kim SH, Suh SB. Tinea capitis of adults in Taegu city for 11 years (1978 1988). Korean J Dermatol 1989;27: 666-671.

5. Kim SL, Lee KC, Jang YH, Lee SJ, Kim DW, Lee WJ, et al. The epidemiology of dermatophyte infection in Southeastern Korea (1979 2013). Ann Dermatol 2016;28:524-527.

6. Cheon SJ, Lee JH, Lee YW, Park J, Suh MK, Kim H, et al. Epidemiology and identification of organisms causing superficial dermatomycoses at tertiary hospitals in Korea: a prospective multicenter study. J Mycol Infect 2018;23:4553.

7. Kim SM, Lee YW, Ahn KJ. A clinical and mycological study of tinea capitis. Korean J Med Mycol 2006;11:184-190.

8. Shin DH, Kim KS, Kim KH. Clinical and mycologic studies of tinea capitis in Taegu. Korean J Med Mycol 1998;3:132138.

9. Chun IK, Lim MH, Lee SC, Won YH. Clinical and mycological studies of tinea capitis in Chonnam area (19861995). Korean J Med Mycol 1996;1:83-89.

10. Kim SM, Kim YA, Cho NJ. A descriptive review of kerion and non-inflammatory tinea capitis. Korean J Dermatol 2019; 57:182-190.

11. Lee WJ, Kim SL, Jang YH, Lee SJ, Kim DW, Bang YJ, et al. Increasing prevalence of trichophyton rubrum identified through an analysis of 115,846 cases over the last 37 years. J Korean Med Sci 2015;30:639-643.

12. Zhan $P$, Liu $W$. The changing face of dermatophytic infections worldwide. Mycopathologia 2017;182:77-86.

13. Lee WJ, Sim HB, Jang YH, Lee SJ, Kim DW, Jun JB, et al. Skin infection due to Trichophyton tonsurans still occurs in people in Korea but not as outbreaks. J Korean Med Sci 2016;31:296-300.

14. Cáceres-Ríos H, Rueda M, Ballona R, Bustamante B. Comparison of terbinafine and griseofulvin in the treatment of tinea capitis. J Am Acad Dermatol 2000;42(1 Pt 1):80-84.

15. López-Gómez S, Del Palacio A, Van Cutsem J, Soledad Cuétara $M$, Iglesias $L$, et al. Itraconazole versus griseofulvin in the treatment of tinea capitis: a double-blind randomized study in children. Int J Dermatol 1994;33:743-747.

16. Jahangir M, Hussain I, UI Hasan M, Haroon TS. A doubleblind, randomized, comparative trial of itraconazole versus terbinafine for 2 weeks in tinea capitis. $\mathrm{Br} J$ Dermatol 1998;139:672-674.

17. Gupta AK, Alexis ME, Raboobee N, Hofstader SL, Lynde $\mathrm{CW}$, Adam $\mathrm{P}$, et al. Itraconazole pulse therapy is effective in the treatment of tinea capitis in children: an open multicentre study. Br J Dermatol 1997;137:251-254.

18. Gan VN, Petruska M, Ginsburg CM. Epidemiology and treatment of tinea capitis: ketoconazole vs. griseofulvin. Pediatr Infect Dis J 1987;6:46-49. 